\title{
Intérêt de la spéciation dans la surveillance biologique de l'exposition professionnelle aux substances inorganiques
}

\author{
Interest of speciation in biological \\ monitoring of occupational exposure \\ to inorganic substances
}

\section{Francis PIERRE}

Laboratoire de Surveillance Biologique, INRS, Avenue de Bourgogne - BP 27 54501 VANDEEUVRE-LÈS-NANCY Cedex - Tél : 0383502043 - Fax : 0383502096

E-mail : francis.pierre@inrs.fr

(Reçu le 10 mai 2001 ; accepté le 20 juin 2001)

\section{RÉSUMÉ}

Les récentes recherches de terrain dans le domaine de la surveillance biologique en milieu industriel ont mis en évidence des particularités cinétiques de l'élimination urinaire de certains indicateurs. Ces résultats remettent en question les préconisations actuelles de valeurs limites pour les indicateurs biologiques d'exposition applicables aux substances inorganiques. L'exemple traité est celui de l'exposition professionnelle à l'aluminium et à ses composés lors de diverses étapes des processus de fabrication et d'utilisation du métal ; la mesure de la concentration de l'aluminium urinaire est utilisée comme indicateur pour assurer le suivi des personnes exposées. L'étude de la cinétique d'élimination urinaire de l'aluminium chez des salariés dans les différentes situations d'exposition a permis de caractériser, premièrement, des niveaux différents d'excrétion urinaire selon la nature physico-chimique du composé d'aluminium présent et deuxièmement, des cycles quotidiens de variation des concentrations dépendant des périodes d'exposition. Le déroulement des phases de ces cycles dans le temps, entre autres la position du maximum de concentration, est spécifique du composé.

Au-delà du cas de l'aluminium, les conséquences en surveillance biologique pour des composés différents dont la

\section{SUMMARY}

Recent field studies in biological monitoring in occupational health highlighted certain kinetic characteristics of biological exposure indices. These results call in the current question again of the limit recommended values for the biological exposure indices applicable to the inorganic substances. Results were obtained in occupational exposure to aluminium and its compounds at the time of various stages of the manufacturing and transformation processes of the metal. The measurement of the concentration of urinary aluminium was used like indicator to ensure the follow-up of the exposed people.

The study of the urinary kinetics of aluminium elimination was conducted in employees in the various exposure situations with licence to characterize, firstly, the levels of urinary excretion according to chemical and physical nature of the aluminium compounds and secondly, the daily cycles of variation of the concentrations dependent on the exposure periods.

The course of the phases of these cycles in time and among others the position of the maximum of concentration is specific to the compound. The consequences in biological monitoring beyond the case of aluminium when the exposure to different compounds is followed by biological measurement 
mesure de l'exposition est assurée par la mesure biologique d'un même élément, touchent les deux paramètres clés que sont le choix d'une valeur limite et la détermination d'un moment de recueil spécifiques.

Cette distinction des espèces chimiques est déjà réalisée pour la surveillance des atmosphères de travail par prélèvement d'aérosols.

\section{MOTS-CLÉS}

Surveillance biologique, spéciation, médecine du travail.

\section{Introduction}

La santé au travail est une préoccupation commune à l'ensemble des pays industrialisés. Afin de prévenir tout effet sur la santé, l'exposition chronique aux substances chimiques doit être évitée ou réduite aux niveaux les plus faibles possible, elle ne doit pas provoquer d'altération de la santé des personnes exposées.

Dans l'entreprise, l'évaluation de l'exposition du salarié est le paramètre essentiel de la prévention du risque chimique. Le médecin du travail est alors l'acteur principal en position de choisir l'indicateur le plus pertinent pour obtenir cette évaluation.

\section{État des lieux}

L'évaluation de l'exposition par mesure des concentrations atmosphériques est le moyen le plus utilisé, elle se réfère à des tableaux de valeurs seuils ayant une valeur réglementaire ou indicative.

Le développement de moyens de surveillance biologique permet en parallèle d'utiliser des indicateurs biologiques d'exposition ou d'effet. Pour chaque substance chimique un paramètre biologique de surveillance est conseillé, assorti d'une concentration à ne pas dépasser. Cette valeur limite non réglementaire est le plus souvent recommandée.

Dans le premier cas pour effectuer la caractérisation et le mesurage des expositions atmosphériques au poste de travail, le médecin du travail peut généralement faire appel aux compétences en prélèvement et analyse de partenaires spécialisés de l'institution prévention. En revanche, pour le choix d'examens relevant de la surveillance biologique il est seul prescripteur.

L'indicateur biologique d'exposition fournit en théorie une information sur la dose interne, qui prend en compte les différentes voies d'apport de la substance, alors que la mesure individuelle de l'exposition ne concerne que la concentration du toxique à proximité des voies respiratoires. L'indicateur biologique est particulièrement utile lorsque le produit est absorbé par la peau, qu'il est ingéré ou que la personne est équipée d'un dis- of the same element touch the two key parameters: the choice for the limit value and the choice for the specific timing of urine collection. This distinction of the chemical species is already operational with regard to the monitoring of the processing atmospheres by dust sampling.

\section{KEY-WORDS}

Biological monitoring, speciation, occupational medicine.

positif de protection respiratoire.

Les listes de valeurs limites atmosphériques (Threshold Limit Values) et biologiques (Biological Exposure Indices) ont été publiées aux USA par l'ACGIH (American Conference of Governmental Industrial Hygienists) $(1,2)$ et en Allemagne par le DFG (Deutsche Forschungsgemeinschaft) (Maximale Arbeitplatz-Konzentration et Biologischer Arbeitsstoff-Toleranz-Wert $(2,3)$. En France, le ministère du travail a publié pour la surveillance des atmosphères des lieux de travail des listes de Valeurs limites de Moyennes d'Exposition (VME) et de Valeurs Limites d'Exposition (VLE) (4) et pour la surveillance biologique les IBE (Indicateurs Biologiques d'Exposition) (5).

La lecture de ces listes permet de constater qu'il existe globalement, en nombre de recommandations, dix fois plus de valeurs limites atmosphériques que de valeurs limites biologiques. Ce décalage correspond en partie à l'apparition plus récente de la surveillance biologique.

\section{Prise en compte de la spé- ciation}

Le deuxième constat concerne les éléments métalliques identifiés sur la liste des valeurs limites atmosphériques.

Sur la liste française par exemple, il apparaît pour l'aluminium $(\mathrm{Al})$ des VME correspondant à six cas différents dont quatre où l'élément aluminium est caracté= risé par ses propriétés physiques ou chimiques. Ainsi sont distingués le métal, le métal finement divisé (pulvérulent), les fumées de soudage et les sels solubles. Dans les deux autres cas, la différenciation porte sur la nature chimique du composé il s'agit du trioxyde de dialuminium et des composés alkylés d'aluminium. A chacune de ces six situations d'exposition identifiées est attribuée une VME spécifique allant de 2 à 10 mg.m-3.

Ces distinctions tendent à identifier de manière spécifique des situations d'exposition en hiérarchisant le 
danger, soit en prenant en compte un risque toxique particulier soit en considérant des différences de biodisponibilité, elles-mêmes liées à des caractéristiques de solubilité ou de granulométrie d'aérosols. L'ensemble de ces distinctions pourrait être considéré comme la prise en compte de la "spéciation" d'un élément considéré, en donnant à ce terme, pour des facilités de langage, une large définition permettant de regrouper des espèces chimiques et des différences physico-chimiques rencontrées pour un élément donné.

Ces considérations de cas particuliers pour un même élément existent pour la plupart des éléments métalliques cités sur la liste, ainsi pour définir l'exposition au "nickel" dix situations d'exposition sont citées.

Sur les listes dédiées aux indicateurs biologiques les paramètres sont nettement moins détaillés, seuls parmi les substances inorganiques, le plomb et le mercure sont envisagés, en Allemagne, sous deux formes chimiques avec prise en compte de composés organiques ; l'arsenic constitue un cas particulier pour lequel il est tenu compte des métabolites afin de déduire l'apport alimentaire.

Autre particularité de ces listes l'existence de paramètres qui ne sont pas des indicateurs d'exposition mais des indicateurs d'effets, tel que le suivi de l'activité acétylcholinestérasique pour l'exposition à des substances inhibitrices de l'activité cholinestérasique.

Il est prévisible que ces listes d'indicateurs biologiques vont progressivement évoluer avec l'apport de compléments dans les trois directions identifiées précédemment, le nombre de paramètres, la prise en compte de la "spéciation" et le développement d'indicateurs d'effets.

\section{Intérêt de la spéciation pour la surveillance biolo- gique}

L'exemple de la nécessité de faire évoluer ces listes peut être tiré des résultats récemment obtenus à l'issue des recherches conduites sur les indicateurs urinaires d'exposition dans les situations d'exposition à l'aluminium en France $(6,7)$.

L'intérêt récent pour la bio-métrologie de l'aluminium est lié aux recherches d'effets neurotoxiques en milieu de travail.

Actuellement, il n'existe pas en France de valeur limite biologique pour l'aluminium. Seule une valeur allemande BAT (2) a été publiée pour la surveillance biologique de cet élément : il s'agit de la mesure de la concentration urinaire en aluminium sur un échantillon de fin de poste, la valeur de concentration à ne pas dépasser est fixée à la valeur très élevée de $200 \mu \mathrm{g} / \mathrm{L}$. Dans l'étude citée, l'importance de la "spéciation" est clairement mise en évidence. Les particularités constatées conduisent à reconsidérer les modalités de recueil de l'échantillon urinaire soumis à l'analyse, donc le protocole de surveillance biologique. Elles permettent également de hiérarchiser les niveaux de concentration atteints. Selon ces résultats une règle unique régissant la surveillance biologique de l'aluminium n'est pas applicable à l'ensemble des situations rencontrées.

Plusieurs entreprises françaises ont participé à l'étude, elles présentaient chacune des situations différentes d'exposition à l'Al ou à ses composés, dans des processus de production et de transformation industriels du métal.

Dans chaque entreprise, des salariés volontaires représentatifs d'expositions particulières ont été suivis durant une semaine selon le même protocole, comportant l'analyse du travail, la mesure durant cinq jours de l'exposition atmosphérique au niveau des voies respiratoires et l'établissement des cinétiques d'élimination urinaire de l'élément aluminium. Chaque cinétique individuelle a été réalisée grâce à la mesure de l'évolution de l'excrétion urinaire de l'aluminium durant sept jours à raison de six à huit recueils d'urine chaque jour. Les substances auxquelles les salariés étaient exposés étaient représentatives des étapes de production de l'aluminium ainsi que de certaines des utilisations du métal.

Ces substances comportant en commun l'élément aluminium étaient les suivantes : le minerai extrait des mines, la bauxite ; les hydroxydes et oxydes, dont l'alumine produite à partir de la bauxite; le fluorure d'aluminium fabriqué à partir de l'alumine ; des composés dont la cryolithe comportant $\mathrm{Al}$ et $\mathrm{F}$ présents lors de la production du métal par électrolyse dans deux situations utilisant des technologies différentes; des composés émis lors des opérations de fusion en fonderie d'aluminium ; la poudre d'aluminium au cours de sa fabrication, ce produit pulvérulent est utilisé, entre autres pour la fabrication du béton cellulaire ; les fines particules d'aluminium produites par les opérations de meulage et de ponçage de pièces coulées en aluminium et les fumées de soudage produites par les procédés de soudage à l'arc.

Dans les situations d'exposition mixte à l'aluminium et aux fluorures les concentrations atmosphériques et urinaires en fluorures ont été également déterminées.

Le recueil en milieu de travail empoussiéré et l'analyse de faibles concentrations d'aluminium ont nécessité l'élaboration de protocoles adaptés afin d'éviter la contamination des échantillons. 


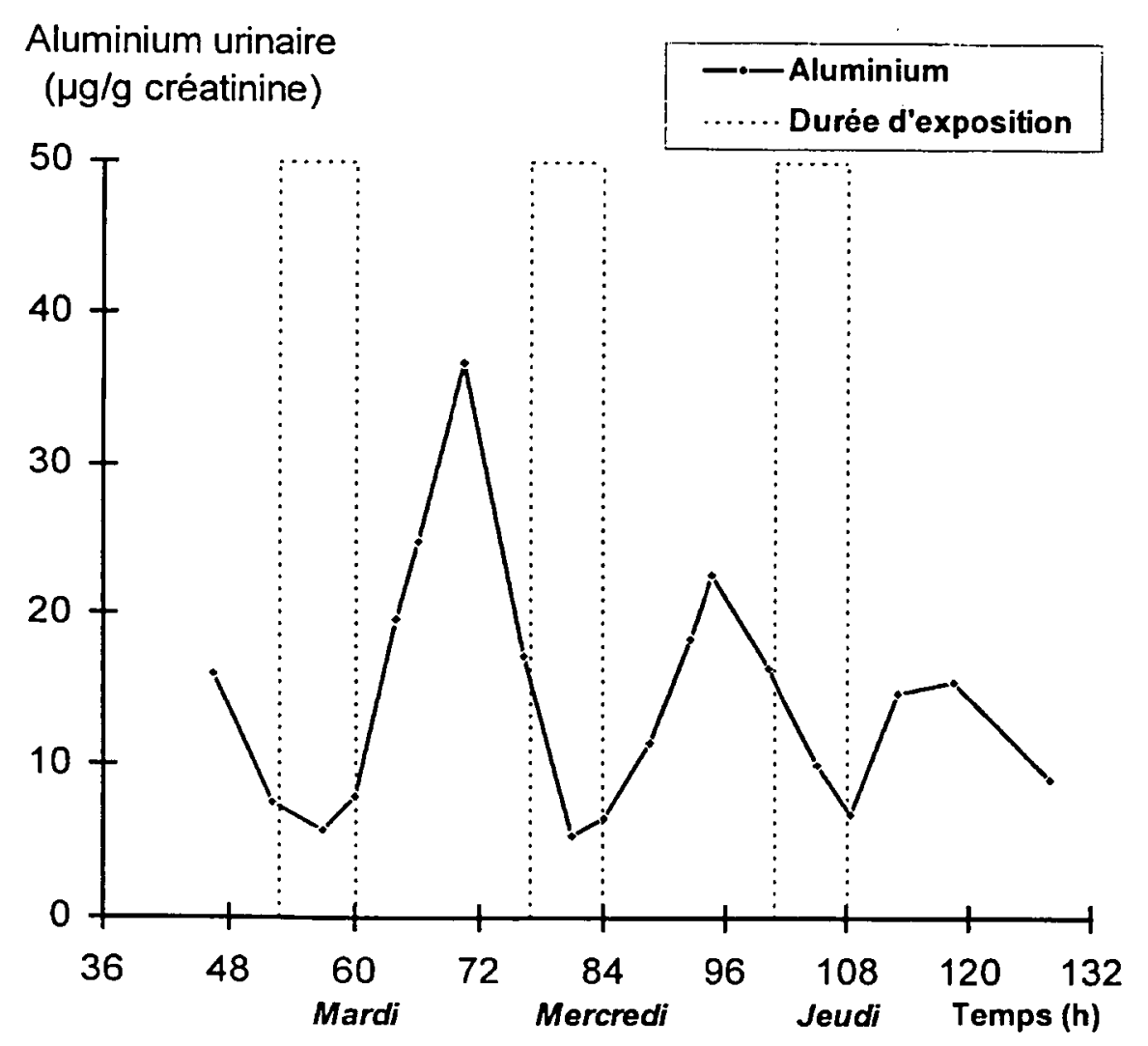

Figure $2 B$ : Évolution de la concentration urinaire d'aluminium durant trois jours de travail. Cas d'un salarié exposé aux poussières de fluorure d'aluminium.

En médecine du travail, en se plaçant dans la pratique actuelle de la prescription d'une analyse d'Al sur un échantillon d'urine, l'échantillon, suivant le protocole habituel, sera prélevé en fin de poste. En fait, selon la nature du composé présent, ce recueil, ne concernera pas la même phase cinétique d'élimination de l'élément Al. Le résultat en concentration urinaire sera donc différent sans que le prescripteur le sache et, en conséquence, l'interprétation du résultat en terme de mesure d'exposition sera pratiquée sur des bases inexactes.

Un cas particulier illustrant l'importance de ces particularités cinétiques, ignorées actuellement dans la pratique, est celui déjà évoqué ci-dessus de l'exposition au fluorure d'Al. Les deux courbes d'évolution des concentrations urinaires en $\mathrm{Al}$ et en fluorures sont présentées sur la figure 3 . La courbe "fluor" présente deux pics en 24 heures; il a été démontré (6) que le premier pic correspond à l'excrétion du fluor due à l'exposition du salarié à l'acide fluorhydrique et le deuxième, moins prononcé, au fluor dû au fluorure d'aluminium (synchronisé d'ailleurs avec le pic $\mathrm{Al}$ dû aussi au fluorure d'Al). Le point remarquable de cette situation est la valeur du déphasage entre les deux courbes aluminium et fluor, ce décalage place pratiquement les deux courbes en opposition de phase. Ainsi, de manière inattendue, un recueil de fin de poste comporte dans le même échantillon la plus faible concentration d'Al et la plus forte concentration de fluorures.

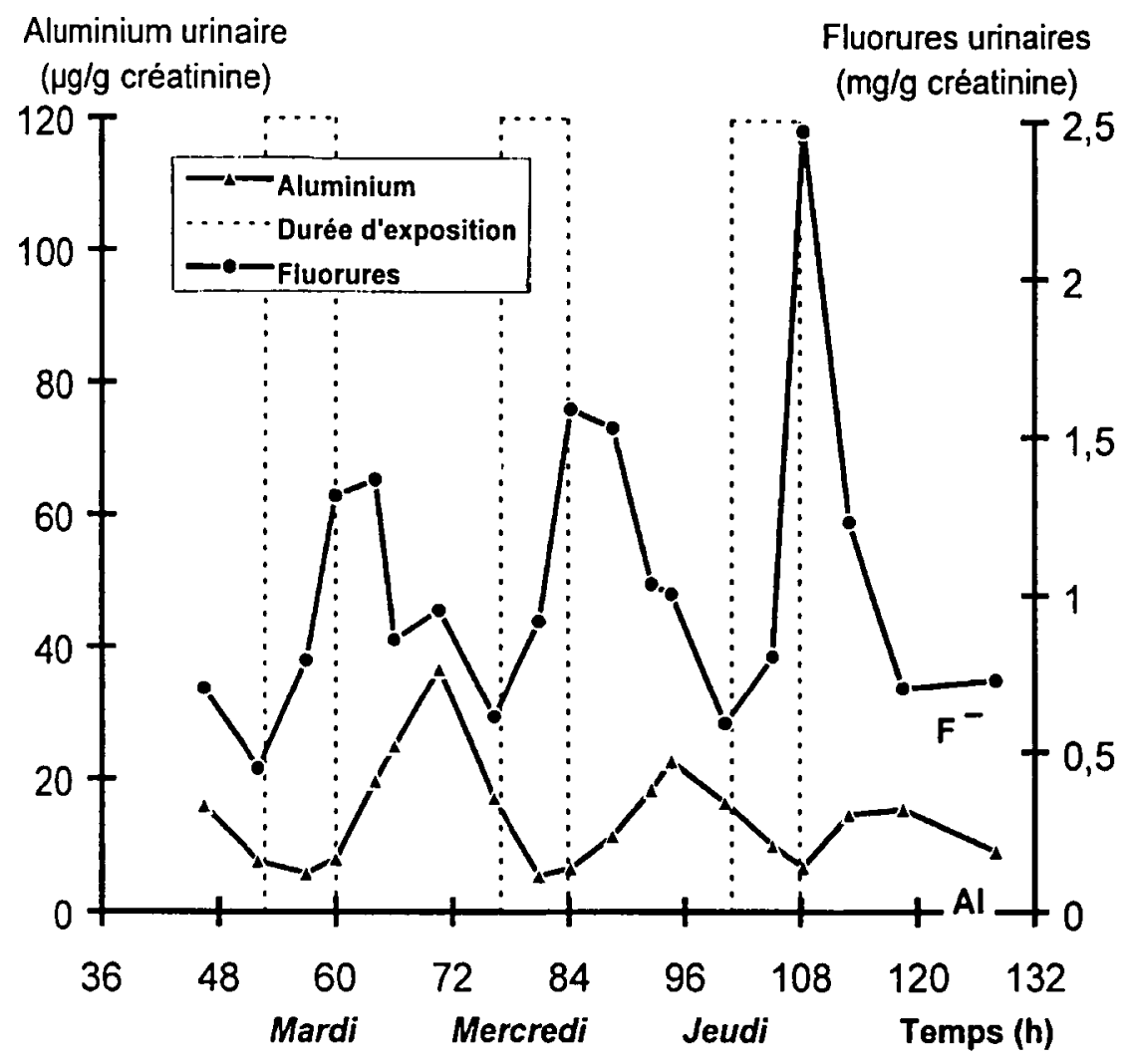

Figure 3 : Évolution de la concentration urinaire d'aluminium et de fluorure durant trois jours de travail. Cas d'un salarié exposé à des vapeurs d'acide fluorhydrique et à des poussières de fluorure d'aluminium.

\section{Discussion}

De l'examen des résultats de l'étude $(6,7)$ il ressort des éléments d'information majeurs sur les effets des différences physico-chimiques ("spéciation"). Ces informations concernent deux aspects de l'influence de la nature du composé d'aluminium, d'une part les niveaux de concentrations urinaires et d'autre part les caractéristiques de la cinétique d'excrétion urinaire.

Les conséquences de ces deux particularités sont à envisager sur deux aspects essentiels du protocole de surveillance biologique de l'aluminium en médecine du travail pour la majorité des sujets.

1) Une exposition à un aérosol d'un composé d'Al donné correspondra à un niveau d'excrétion variable fluctuant selon l'intensité de l'exposition, la fluctuation attendue sera comprise dans un domaine de concentrations spécifique du type d'exposition.

Pour les différents groupes étudiés, cette spécificité conduit à l'existence d'un gradient de ces domaines de concentration, si un risque toxique est lié à l'indicateur, le gradient de concentration identifie des groupes à risque croissant. Dans ce cas la même valeur limite pour l'Al urinaire est applicable à toutes les situations d'exposition.

En revanche, dans l'hypothèse où un risque toxique est lié non pas à la seule concentration de l'élément $\mathrm{Al}$ mais à la nature du composé, la même règle n'est plus applicable à toutes les situations. Dans cette hypothèse, 
il devient nécessaire pour établir une surveillance biologique basée sur l'utilisation du même indicateur, d'identifier une limite spécifique pour chaque domaine d'exposition.

2) En fonction des données ci-dessus et quelle que soit l'hypothèse retenue, le résultat biologique doit fournir la plus grande pertinence en terme de reproductibilité et de significativité. Les spécificités cinétiques sont apparues comme étant des paramètres majeurs expliquant la variabilité de la concentration urinaire. De ce fait, la pertinence de l'indicateur a été clairement trouvée dépendante du choix du meilleur moment au cours de la semaine pour effectuer le recueil de l'échantillon d'urine à demander au salarié.

La poursuite de l'étude sur l'aluminium devrait aboutir à des propositions plus spécifiques à chaque cas, la même démarche devrait être engagée pour les composés d'autres d'éléments inorganiques.

\section{Conclusion}

La confirmation de ces particularités pourrait conduire à adopter, non plus une seule valeur limite pour l'aluminium, mais des valeurs limites spécifiques pour chaque composé, afin de protéger les personnes exposées dans les différentes situations d'exposition. Cette approche pourrait être étendue à d'autres polluants.

L'existence de retard spécifique d'excrétion apparaît être un facteur essentiel de compréhension des mécanismes cinétiques d'excrétion. Jusqu'à présent, les études de terrain, réalisées en grande majorité uniquement avec des échantillons biologiques ponctuels, n'étaient pas en mesure d'aborder cette nécessaire dimension cinétique de l'excrétion urinaire. D'autre part, les études de modélisation ne considéraient pas ce paramètre dans leurs modèles physiologiques.

D'une manière plus générale, une plus grande prise en compte de la nature exacte de la substance polluante apparaît maintenant nécessaire pour garantir l'efficacité d'un protocole de surveillance biologique. Cette évolution de la discipline devrait comprendre une meilleure connaissance des modalités d'excrétion en situation réelle.

\section{Références}

1. American Conference of Governmental Industrial Hygienists - Threshold Limit Values and Biological Exposure Indices for 2000-2001.

2. Schneider O., Brondeau M.T. Indices biologiques d'exposition. Notes Documentaires, INRS, 2099-174-99.

3. Deutsche Forschungsgemeinschaft. List of MAK and BAT Values 2000. Maximum Concentrations at the Workplace and Biological Tolerance Values for Working Materials. Commission for the Investigation of Health Hazards of Chemical Compounds in the Work Area.

4. Valeurs limites d'exposition professionnelle aux agents chimiques en France. Cahiers de Notes Documentaires, INRS, $\mathrm{n}^{\circ}$ 174, 1999.

5. Indices biologiques d'exposition. Principes de base et valeurs-guides utilisables en France. Cahiers de Notes Documentaires, INRS, $\mathrm{n}^{\circ}$ 151, 1993.

6. Pierre F., Baruthio F., Diebold F., Biette P. Effect of different exposure compounds on urinary kinetics of aluminium and fluoride in industrially exposed workers. Occupational and Environmental Medicine, 1995 : 396403.

7. Pierre F. Biomonitoring of Aluminium in Production Workers. Health in the Aluminium Industry. In Managing Health Issues in the Aluminium Industry, $1998: 68-89$. Ed. N.D. Priest and T.V. O'Donnell. Middlesese University Press. London, England. 